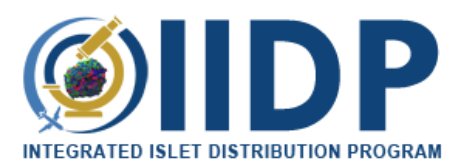

\section{VERSION 1}

\section{JUL 15, 2020}

\section{open $\partial$ ACcess}

DOI:

dx.doi.org/10.17504/protocol s.io. bhcmj2u6

\section{External link:}

https://iidp.coh.org/lnvestigato rs/Policies-Standard-OperatingProcedures

Protocol Citation: Integrated Islet Distribution Program 2020. Flash Freezing Unclaimed Islets. protocols.io https://dx.doi.org/10.17504/p rotocols. io. bhcmj2u6

License: This is an open access protocol distributed under the terms of the Creative Commons Attribution License, which permits unrestricted use, distribution, and reproduction in any medium, provided the original author and source are credited

Protocol status: Working We use this protocol and it's working

Created: Jun 09, 2020

Last Modified: Jul 15, 2020

PROTOCOL integer ID: 37997

\section{(3) Flash Freezing Unclaimed Islets V.1}

\author{
Integrated Islet Distribution Program ${ }^{1}$
}

${ }^{1}$ Integrated Islet Distribution Program, City of Hope

Integrated Islet Distribution Program

Tech. support email: iidp-email@coh.org

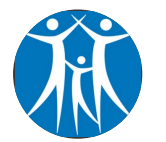

Integrated Islet Distribution Program

Integrated Islet Distribution Program, City of Hope

\section{ABSTRACT}

The objective of the Integrated Islet Distribution Program (IIDP) is to develop a standardized procedure for the freezing and storage of unclaimed islet tissue to approved investigators for use in the National Institute of Diabetes and Digestive and Kidney Diseases (NIDDK) sponsored research in the IIDP. This SOP replaces the IIDP SOP: SHP-002 Flash Freezing Unclaimed Islets.

This protocol provides an opportunity for the isolating centers to recoup expenses for isolated islets that cannot be placed through the normal distribution system at the time of isolation. It also may provide a less expensive and more advantageous method of islet supply to investigators that need non-viable islet tissue for gene panel research, RNA expression, and other experiments where viable islets are not necessary. This protocol also allows the storage of islet tissue for supplemental experiments that may be realized at a later date, after the original distribution has been completed.

\section{Note}

\section{Integrated Islet Distribution Program (IIDP) (RRID:SCR_014387)}

\section{GUIDELINES}

\section{Responsibilities}

- It is the responsibility of the IIDP Coordinating Center (CC) to both follow and ensure adherence to the procedures outlined in this SOP. In order to accomplish this, the IIDP CC will interact with the relevant personnel from each of the participating centers.

- It is the responsibility of each IIDP center to follow the procedures listed in this 
Keywords: flash frozen, human islets
SOP and to work to the best of their ability to follow all requirements.

- It is the responsibility of each approved investigator receiving flash frozen (FF) islets through the IIDP to cooperate in providing feedback to the IIDP on the condition of the FF islets upon receipt and to reuse and recycle all shipping materials to the best of their ability.

\section{Definitions}

- Integrated Islet Distribution Program (IIDP) (RRID:SCR_014387): The IIDP is a program funded by the NIDDK to provide quality human islets to the diabetes research community to advance scientific discoveries and translational medicine. The IIDP consists of the NIDDK Project Officer (PO), the External Evaluation Committee (EEC) and the IIDP Coordinating Center (CC) at City of Hope (COH). The IIDP CC integrates an interactive group of academic laboratories including the subcontracted IIDP Islet Isolation Centers (IIC) and the Human Islet Phenotyping Program (HIPP).

- IIDP Coordinating Center (CC): Joyce Niland, Ph.D. is the Principal Investigator for the IIDP CC and leads staff from the Department of Diabetes and Cancer Discovery Science, Diabetes and Metabolism Research Institute at $\mathrm{COH}$ to coordinate the activities of the IIDP and assist the participating centers and investigators in the distribution of human islets.

- Islet Equivalent (IEQ): A conversion factor of the size of an actual islet to an equivalent size of an islet which is $150 \mu \mathrm{m}$ diameter by mathematically compensating for the volume.

- Approved Investigators: Researchers who have requested islets from the IIDP for basic science studies and whose research protocols have been reviewed and approved by the IIDP.

- Islet Allocation System (IA): This is the online system administered by the IIDP to allow fair distribution of basic science islets to approved investigators. This interactive system is used by the IIDP centers and the approved investigators facilitates and tracks the distribution of human islets. 
MATERIALS

8 Corning $^{\text {TM }}$ Hanks Balanced Salt Solutions (HBSS) or equivalent Fisher

Scientific Catalog \#MT21020CM

\section{Equipment}

\section{Long-Term Storage Cryogenic Tubes or equivalent}

NAME

Cryogenic Tubes - $2 \mathrm{~mL}$

Thermo Scientific $^{\mathrm{TM}}$ Nalgene ${ }^{\mathrm{TM}}$

https://www.fishersci.com/shop/products/nalgene-general-long-termstorage-cryogenic-tubes/033377d

Sterile, externally threaded Thermo Scientific ${ }^{\mathrm{TM}}$ Nalgene $^{\mathrm{TM}}$ General Long-Term Storage Cryogenic Tubes, ideal for research and general lab storage. 


\title{
Safety information
}

Carefully place cryogenic tubes in a liquid nitrogen bath taking all safety precautions to protect you from being burned by liquid nitrogen (mask, gloves, tongs, and apron).

\section{Safety information}

\begin{abstract}
Alert:
Cryogenic vials are intended for placement only in the vapor phase of liquid nitrogen and should not be used for storage in the liquid phase of liquid nitrogen. Immersion of the vials in the liquid phase could result in penetration of the liquefied gas into the vial, resulting in rapid vaporization of the liquid upon removal and possible explosion or leakage from the vial/closure perimeter.

To prevent cryogenic vials from exploding, never overfill liquid nitrogen storage units. Always examine vials before use to ensure no visible defects around the closure rims. Always use full face shields, heavy safety gloves and laboratory protective apparel when removing vials from cryogenic storage. Always permit vials to warm slowly in a biological safety cabinet. Never reuse cryogenic vials.
\end{abstract}

\section{BEFORE START INSTRUCTIONS}

\section{References:}

Scharp DW, Arulmoli J, Morgan K, Sunshine H, Hao E: Advances in Human Islet Processing: Manufacturing Steps to Achieve Predictable Islet Outcomes from

Research Pancreases. OBM Transplantation 2019 Feb, Volume 3, Issue 1 (Current Advancement of Islet Cell Transplantation in the Treatment of Diabetes Mellitus).

\section{Materials}


The following supplies are necessary for the preparation of human islets prior to flash freezing for distribution.

- Islet preparation and distribution

- Sterile pipettes, pipettors, and other appropriate sterile labware for manipulation of islet preparations.

- Hanks Buffered Salt Solution (HBSS) - no additives.

(Note: Either HBSS with or without Calcium, Magnesium, and phenol red can be used.)

- $15 \mathrm{~mL}, 50 \mathrm{~mL}$, or $250 \mathrm{~mL}$ conical tubes (depending on final volume of islet prep).

- Thermo Scientific ${ }^{\text {TM }}$ Nalgene ${ }^{\text {TM }}$ General Long-Term Storage Cryogenic Tubes Self- Standing with Round Bottom (Product \#03-337-7D) (This brand and style of cryogenic tubes are recommended but not mandatory)

\section{Safety information}

\section{Alert:}

Cryogenic vials are intended for placement only in the vapor phase of liquid nitrogen and should not be used for storage in the liquid phase of liquid nitrogen. Immersion of the vials in the liquid phase could result in penetration of the liquefied gas into the vial, resulting in rapid vaporization of the liquid upon removal and possible explosion or leakage from the vial/closure perimeter.

To prevent cryogenic vials from exploding, never overfill liquid nitrogen storage units. Always examine vials before use to ensure no visible defects around the closure rims. Always use full face shields, heavy safety gloves and laboratory protective apparel when removing vials from cryogenic storage. Always permit vials to warm slowly in a biological safety cabinet. Never reuse cryogenic vials.

- Cryogenic markers or pencils for tube labeling.

- Centrifuge with capabilities of spinning the appropriately sized conicals and the $2 \mathrm{~mL}$ cryogenic vials at $1000 \mathrm{rpm}$ for 2 minutes.

- Sterile pipettes, pipettors, and other appropriate sterile labware for manipulation of islet 
preparations.

- Cryo Storage Log sheet

- IIDP Frozen Box Labels from IIDP template.

- Flash Frozen Tissue Shipment Forms.

- FedEx Shipping Form.

- Liquid nitrogen and appropriate vessels and equipment for safely freezing the unclaimed islets.

- Liquid nitrogen storage tanks or $8^{\circ}-80^{\circ} \mathrm{C}$ or $8^{\circ}-135^{\circ} \mathrm{C}$ freezer

- Appropriately sized Styrofoam shipping boxes and adequate dry ice (at least $5 \mathrm{lbs}$ ).

\section{Islet Isolation and Distribution}

2 Perform islet isolation, followed by culture (Islet Culture and Preparation for Cold Shipping SOP) and the distribution of available islets through the IIDP islet allocation system (IA).

\section{Flash Freezing of Unclaimed Islets:}

3 At the end of the islet distribution final offer deadline, if there are still unclaimed cultured islets of greater than $80 \%$ purity, 1 to 10 aliquots of approximately 1,000 IEQ may be flash frozen and stored for future distribution using the following method:

4 Calculate the necessary volume of the islet suspension needed to provide approximately 1,000 IEQ per appropriately sized conical tube and divide left over islets needing to be frozen into the required number of tubes.

5 Label the appropriate number of cryogenic tubes with Isolation \#, RRID, and date using a cryogenic marker or pencil and set aside. All tubes should have a final aliquot of approximately 1,000 IEQ. 
Centrifuge conical tubes containing the islets at $\because 1000 \mathrm{rpm}$ for 2 minutes at $4^{\circ} \mathrm{C}$.

7 Carefully evacuate supernatant from the islet pellet.

8 Add $1 \mathrm{~mL}$ of serum free HBSS to the conical tube in order to dilute any serum from the remaining culture media. Gently resuspend the islet pellet and transfer islet suspension to a $2 \mathrm{~mL}$ labeled cryogenic tube. Add another $0.5 \mathrm{~mL}$ of HBSS to conical and rinse to capture remaining islets. Transfer to the cryogenic tube. Rinse with $0.5 \mathrm{~mL}$ HBSS. Repeat for all tubes to be frozen.

$9 \quad$ Centrifuge cryogenic tube at $\because 1000 \mathrm{rpm}$ for 2 minutes.

10 Evacuate supernatant, leaving a dry pellet.

11

\section{Safety information}

Carefully place cryogenic tubes in a liquid nitrogen bath taking all safety precautions to protect you from being burned by liquid nitrogen (mask, gloves, tongs, and apron)

\section{Safety information}

Safely transfer the vials into a labeled container and store in a $8^{\circ}-80^{\circ} \mathrm{C}$ or $8^{\circ}-135^{\circ} \mathrm{C}$ freezer or in the vapor phase of a liquid nitrogen storage tank until shipment. 


\section{Cataloguing of Frozen Islets:}

13 Complete the first six columns of the IIDP Cryo Storage Log Sheet. A separate line should be used for each tube frozen.

\section{Attachment 1-Cryo Storage Log.xlsx}

Note

This Cryo Storage log sheet is made available for in-house use and center convenience (It does not have to be returned to the IIDP)

14 The log sheet should be updated as the islet aliquots are removed.

\section{Broadcast of Frozen Aliquots:}

15 The flash frozen islets can be broadcasted at any time after the original isolation has been broadcasted and distributed and islets with greater than $80 \%$ purity remain. The broadcast is not time dependent and interested investigators can claim these islets at any time after broadcast until all have been distributed.

16 Log into the IIDP database using the appropriate ID and Password.

17 Log into the Dashboard, click on "Frozen Islets" on the Navigation Menu on the left hand side of the page. Click on "Broadcast Frozen Islet Offer". 
18 When the center's list of isolations displays, click on the "Broadcast" button of the isolation for intended broadcast of the flash frozen islets. A new screen will prompt a few questions about the timing of when the islets were frozen, how many vials are available (the total number of islets will automatically be populated at 1,000 IEQs per vial) and the purity of the frozen samples (the purity must be greater than $80 \%$ to be useful as flash frozen samples).

19 Once the form is completed, click on "Broadcast" and the information will be entered into the database system. At this point an email will be sent to all possible investigators. A confirmation notice will display that the broadcast has been successful.

20 The investigators will then enter the system and, if interested, will accept the offers. (This is similar to the cultured islet broadcasts but the flash frozen islets are not time dependent.)

21 The broadcasting center will be alerted by email when the flash frozen preparations have been accepted. At this point, the center staff should go back into the system to the "All Frozen Islet Offers" and look for "New Request" under the column "Frozen Islets Accepted " indicating an accepted offer.

Note

Note: Older isolations may have aliquots of 5,000 or 10,000 IEQs.

22 To ship the Flash Frozen aliquot, the center staff should click on the red "Confirm Shipment" button on the row that has the "New Request" indicator. The "Confirm Recipients" screen will appear and should be completed by center staff. Click Edit to insert the tracking number and the amount being shipped. After submission, the "Inventory List" will be populated with the appropriate information and emails will be sent to notify acceptance by the center staff with the shipping information to both the investigators and the center staff. Once a recipient is confirmed, click on the "Print Tissue Shipment Forms" and include the appropriate form with each shipment.

Note

Note: The center must click on the Print Tissue Shipment Form (TSF) in order to complete the distribution process and trigger the email distribution. 


\section{Shipping of Frozen Aliquots:}

23

Note

Islets should be maintained below $8^{\circ}-80^{\circ} \mathrm{C}$ until ready for shipment.

A completed Flash Frozen Tissue Shipment Form should accompany each sample shipment. Approximately five pounds of dry ice should fill a small, thick Styrofoam shipping box. Multiple tubes of 1,000-10,000 IEQ can be sent in a single box if necessary to satisfy the number of requested islets. The tubes should be placed in the middle of the dry ice. The IIDP Frozen Sample Labels should be placed on two sides of the box and the dry ice regulation label should be completed before shipping.

24 Samples should be sent priority overnight shipping by FedEx with appropriate boxes checked on the FedEx form for dry ice shipments. (No materials will be returned by the investigators.)

25 User Feed Back Forms should be completed online by the investigators. 